\title{
OPTIMISATION OF LARGE SCALE MAINTENANCE NETWORKS WITH EVOLUTIONARY PROGRAMIIING
}

\author{
KOTA, L.
}

Abstract: This study develops a single phase algorithm for the fixed destination multi-depot multiple traveling salesman problem (MD-MTSP) with sub tour construction. First show the general model of the technical inspection and maintenance systems, where this problem usually emerges and present the most important participants in these systems, like the virtual logistic centres, experts, objects, repair companies, warehouses, suppliers. It propose a mathematical model of the system's object expert assignment with the constraints like experts minimum and maximum capacity, constraint on expert maximum and daily tour. In the next part it presents the developed evolutionary programming algorithm which solves the assignment, regarding the constraints with the usage of penalty functions in the algorithm. In the last part of the paper the convergence of the algorithm and the solving times are presented.

Key words: evolutionary programming, heuristics, logistics, maintenance networks
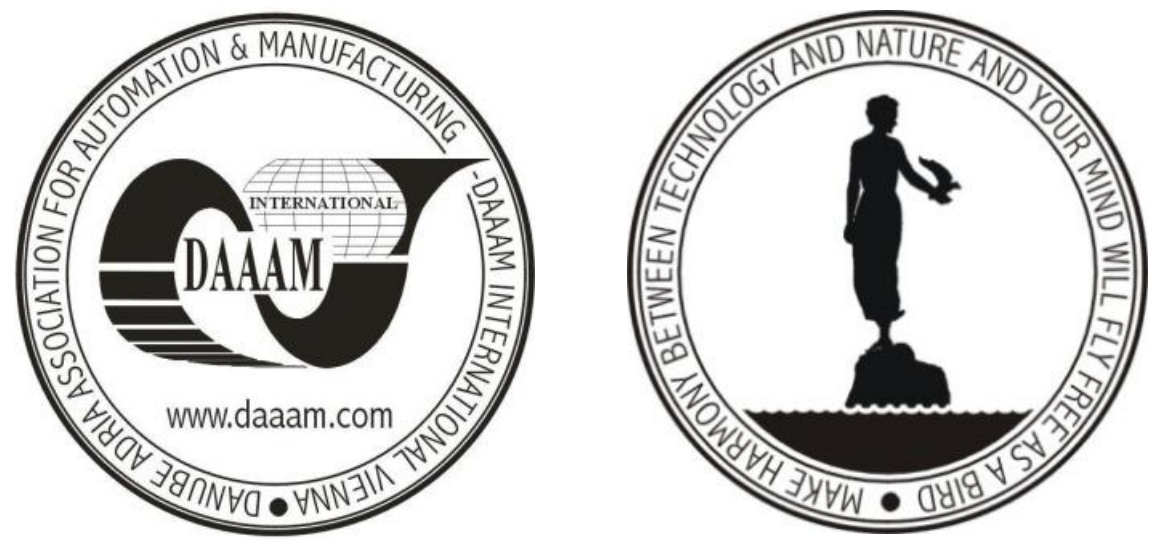

Authors' data: Kota, L[aszlo], University of Miskolc, Miskolc-Egyetemvaros, 3515, Miskolc, Hungary, altkota@uni-miskolc.hu

This Publication has to be referred as: Kota L[aszlo] (2011). Optimisation of Large Scale Maintenance Networks with Evolutionary Programming, Chapter 40 in DAAAM International Scientific Book 2011, pp. 495-512, B. Katalinic (Ed.), Published by DAAAM International, ISBN 978-3-901509-84-1, ISSN 1726-9687, Vienna, Austria

DOI: $10.2507 /$ daaam.scibook.2011.40 


\section{Introduction}

In these days in the field of globalized production and service industry the significance of the tightly logistics integrated systems are increasing. While in the beginning mostly the production industry gets globalised, nowadays there are multinational companies which offer even world spread service solutions.

In the service industry the technical inspection and maintenance systems has emphasized importance because they provide the safe and solid operation of production or service facilities. Maybe most significant facilities are the communal services, water supply, electricity, district heating, fuel supply, telecommunication services or even elevators found in residential areas in large numbers.

The reliable, accident free, and economic operation require periodic technical inspections and maintenances. In these systems the inspection generally require specialized knowledge, sometimes it even requires special certificate. Like for example the elevators which inspection and maintenance are very important from the aspect of life protection, according to this these field are regulated by governmental ordinances.

There could be similarly treated the devices which requires periodic inspection and maintenance, for example the safety and control devices of the electricity, gas, heat, water supply networks, monitoring devices, critical network control devices which require on site supervision and maintenance.

In these networks the following tasks emerges: (1) an maintainer person (called as expert hereafter) have go to the site several times in a year and there do the inspection and/or maintenance duties, (2) the maintenance and inspection tasks requires different tools and parts which has to transport to the site and/or back to the warehouse on time, (3) the experts have to reside near the objects to reach lesser time expenditure and cost, (4) the required materials stored several in warehouses scattered in the system, (5) when an onsite non-repairable part emerge the parts have to transfer to a repair/refurbishment facility

The main problem is in these type of systems is to assign the experts to the objects what they inspect and control them on everyday route while the expert have to inspect the objects and have to return to his home location at the end of the day beside with optimal number of expert to reduce the costs. This paper gives or tries to give a solution - even if some details not mentioned here due to lack of space - a heuristic optimisation to this problem, even in large scale systems.

\section{System model of the network like inspection and maintenance systems}

The network like technical inspection and maintenance systems can extend a city, a region, a country, continent wide, or even worldwide. The duties of these systems are a regular supervision of the objects in a defined time period and maintenance and/or repair the parts of the objects. The effective realizations of the maintenance tasks is ensure by one or more scattered raw material and tool warehouses and repair facilities. 
The role of the logistic system is to ensure the availability of the resources experts, raw materials, tools- required by the technical inspection and maintenance tasks

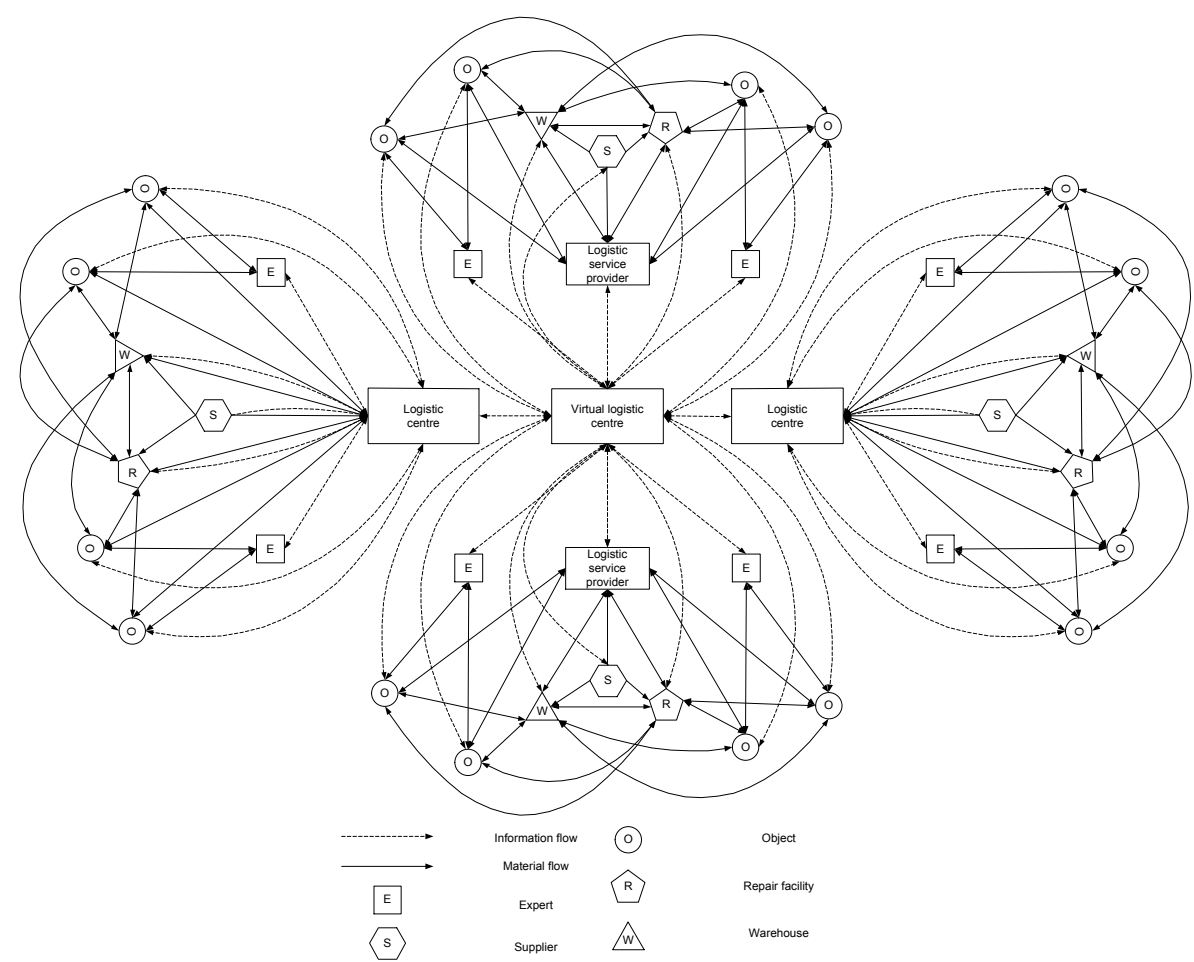

Fig. 1. General structure of a technical inspection and maintenance system

Regarding the geographical scatter of the required resources - experts, materials, tools - and demands - technical inspection and maintenance -, the optimal operation of the system have to ensure by a virtual logistic centre or a company with a logistics centre where all the material and information flow centred and where the whole system controlled.

The system is controlled by a virtual logistic centre (Fig. 1.), but in smaller scale - regional or country wide systems - the core of the system, the controller facility could be a logistic centre where the information processing and the material flow is simultaneously present. The virtual logistic centre is in information link with all of the system components, namely the: (1) experts, (2) warehouses, (3) repair facilities, (4) suppliers,(5) logistic service providers, which provide the handling of transfer tasks in the system.

The virtual logistic centre is not involved in the physical material flow; it only controls it and allocates the resources. The tasks of the virtual logistic centre: (1) decide which expert is chosen to the system, choose its location, (2) assign the experts to the objects, (3) schedule and register the required technical inspections and maintenances, (4) choose the location of the warehouses, repair facilities, (5) choose the suppliers, (6) dispose and schedule transports to the warehouses and repair facilities, (7) allocate the vehicles of transportations.

The virtual logistic centre which controls the system uses complex mathematical models and optimisation processes, where it minds the operational requirements, governmental regulations and many other conditions as constrains. 


\section{System components and their functions}

\subsection{Objects}

The objects are such machines facilities which: (1) require $\kappa_{i(i=1 . . p)}-$ where $p$ is the number of the objects - maintenances in a $\vartheta$ time period, depends on the age or technical condition or other regulations. (2)The inspections can be done with an optional interval but it can't be too short. (3) At all of the objects all the maintenances are mandatory, in some cases it is defined by law.

\subsection{Experts}

In most cases they are specialized technical experts, who do the $\kappa_{i}$ technical inspections in the given $\vartheta$ period. Their role could be according to the type of the system: (1) only technical inspection, supervision or control test, (2) do some smaller maintenance tasks, (3) control and supervise and approve bigger maintenance tasks.

\subsection{Repair facilities}

Most of the inspection and maintenance tasks are performed onsite by the experts occasionally with technicians of the repair company. In the repair facilities such tasks are performed which cannot do onsite. The need of a repair facility primarily appears at repairing large parts, or complex and long period repair tasks. The tasks of a repair facility: (1) repair of the supplied parts, (2) refurbish of the supplied parts, (3) recycle of the supplied parts and sometimes, (4) sell of the refurbished parts or machines. The repair facility did not do any transportation tasks. All the transportation done by the logistic service provider, or sometimes the experts can transport smaller parts, tools. At the design of complex machines one of the design targets is the modularity, so the machines can be maintain and repair fast with swapping the defective module with a new, and therefore the downtime decreased to the minimum and the defective module could be repaired - which is a long task in time - or recycled at the repair facility.

\subsection{Warehouses}

The role of the warehouses is storing: (1) the spare parts, (2) the refurbished parts, (3) some transportation vehicles, (4) auxiliary materials, consumables, tools, etc.

It is important to uphold such a stock level which minimizes the downtime caused by the maintenance.

\subsection{Suppliers}

The role of the suppliers is: (1) deliver the spare parts to the warehouses, (2) deliver the tools, consumables to the repair facilities, (3) and deliver the tools and consumables to the warehouses.

In these systems the transportation could be the task of: (1) the supplier, (2) an external forwarder company, (3) the logistic centre.

Selecting the suppliers belongs to the main tasks of the virtual logistic centre. 


\subsection{Logistics service providers}

The tasks of the external logistics service providers are to fulfil the tasks of transportation, storage, or other complex logistic operations like finishing. Distinguishing by their activities there is: (1) logistic service company: it does only transportation and storage tasks (2) logistic centre: it does complex logistic services beside the basic operations the storage and transportation

The transportation tasks are: (1) transport spare parts and tools from the warehouses to the objects, (2) if the suppliers does not transport the spare parts on their own, transport the spare parts from the suppliers to the warehouses, (3) transport the supplied material to the repair facilities if the suppliers does not do, (4) transport the repaired and refurbished parts from the repair facilities to the warehouses.

If the logistic service providers do any storage tasks then the role of the warehouses are also applied (chapter 3.4). The storing at the logistic service providers does not exclude the usage of dedicated warehouses near the sites.

The logistic centre could do the transportation, storage or even local disposition tasks performing complex logistic services. Deploy or involve a logistic centre into the system generally happen on regional level, because their scope of services is so extensive they can substitute warehouses and logistic service companies on regional level or even can perform some disposition and management tasks of the virtual logistic centre.

\subsection{Virtual logistics centre}

The virtual logistic centre is the core of system. The virtual logistic centre manages the operation of the system and does the following tasks: (1) select and allocate and assign the required logistic resources, (2) collect and process the data which required to the selection and the assignment of the optimal network elements, (3) operate the information system to which all the system elements linked, (4) define the management structure of the system and operate the logistic system, (5) collect and store the data generated by the logistic networks and its connected elements which needs to the system benchmarks and performance management, (6) constantly monitoring on the performance and decide which element can outsourced and examine the possibilities of forming regional centres.

\section{Mathematical model of the technical inspection and maintenance systems}

The system main parameter is the path matrix, which shows the distance of the system elements. In our case the path matrix is an integrated matrix, it builds up from several sub matrixes, the sub matrix count defined by the number of elements in the system.

$$
L=\left.\left[l_{i j}\right]\right|_{i, j=1 . . q}
$$




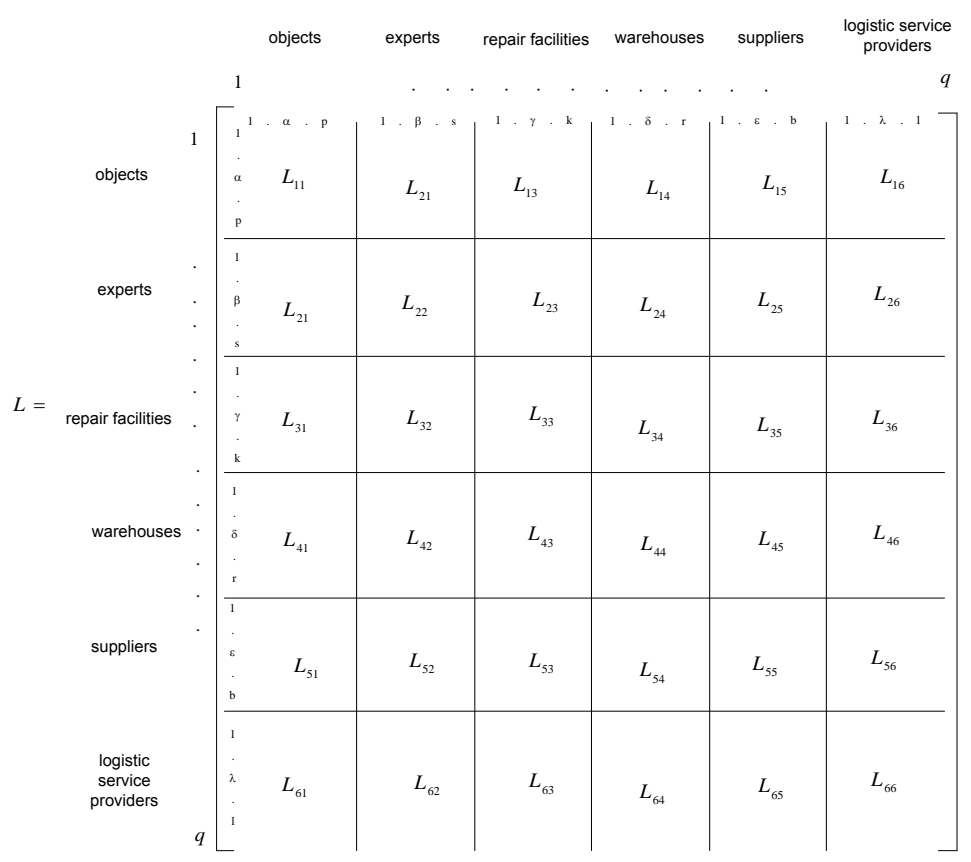

Fig. 2. Path matrix

where: (1) $q=p+s+k+r+b+l$ : the size of the matrix, (2) $p$ : the number of the objects, (3) $s$ : the number of the experts, (4) $k$ : the number of the repair facilities (5) $r$ : the number of the warehouses, (6) $b$ : the number oft he suppliers, (7) $l$ : the number of the logistic service providers.

The partitions of the path matrix are generally symmetrical, but in some cases when the distance is not the same in the backward direction the path matrix asymmetrical. This happens mostly at inner city routes where a lot of one-way street are.

The assignment matrix is one of the main output parameters of the model. The structure of the assignment matrix is the same - contains the same sub matrix structure - as the path matrix, it shows the connection between the system elements.

$$
Y=\left.\left[y_{i j}\right]\right|_{i, j=1 . . q}
$$

where:

- $y=\left\{\begin{array}{l}1 \\ 0\end{array}\right.$ according to the system elements are assigned to together (1) or not (0),

- $q=p+s+k+r+b+l$, is the size of the matrix.

Defining the $y_{i j}$ matrix is the assignment task which have to solved in this complex system.

\subsection{Objects}

The main parameters of the objects are: (1) $p$ : is the number of the objects, it could be constant or dynamically changed, (2) L matrix defines the location of the objects, and the distance from the other system elements, (3) $\kappa_{i(i=1 . . p)}$ is the mandatory inspection per object, (4) $M T B F_{i}$ : the mean time between the failures at 
the object $\mathrm{i} \varepsilon_{i(i=1 . p)}$, from thos parameter one can calculate the required ad hoc maintenance tasks, (5) $\tau_{i}^{K}{ }_{(i=1 . p)}$ is the average time of a technical inspection, maintenance on the object $i$.

$$
\varepsilon_{i}=\frac{\vartheta}{M T B F_{i}} ; \varepsilon=\left[\varepsilon_{i}\right]_{i=1 . . p}
$$

The number of the technical inspections and maintenances could be prescribed by law, governmental regulations in some cases where human life is endangered, like the elevators. The maintenances can't happen in a arbitrary period, there is a time period which have to be defined to every object when the next maintenance task couldn't performed and the interval of the inspections fulfil the

$$
\tau^{m}=\left[\tau_{i}^{m}\right]_{i=1 . . p} ; \tau_{i}^{m} *\left(\varepsilon_{i}-1\right) \leq \vartheta
$$

constrain. In real conditions in these systems the inspection and maintenance tasks are performed by the same expert so the special knowledge collected at the previous inspections is well utilized so the maintenance times could be shortened.

\subsection{Experts}

The parameters for the mathematical description of the experts are the following: (1) $s$ : is the number of the experts, constant in most cases, but it could change for example at the case of: (a) expert leaving the job, (b) expert employment in case of system expansion, (c) expert number decreasing in case of system reduction it could be dynamic. (2) $L$ matrix: defines the location of the expert, well it defines the distance of the experts from the other system elements, (3) $\bar{v}$ : the average speed of the expert, generally it defined as constant.

From these parameters the visiting time of the object $i$ at the expert $h$ can be defined as:

$$
\tau_{i}^{f}=\frac{l_{p+h, i}}{\bar{v}}
$$

where:

- $l_{p+h, i}:$ is the distance between the object $\mathrm{i}$ and expert $\mathrm{h}$.

The time required to travel between object $\mathrm{i}$ and $\mathrm{j}$ :

$$
\tau_{i, j}=\frac{l_{i, j}}{\bar{v}}
$$

where: (1) $l_{i, j}$ : is the distance between the object $\mathrm{i}$ and $\mathrm{j}$, (2) $P$ : is the performance of the experts, it show how much maintenance task is performed by the expert.

Constrains:

The performance of the expert has to be between the defined minimum and maximum values: 


$$
P_{i \min }<P_{i}<P_{i \max }
$$

where

$$
P_{i}=\sum_{j=1}^{p}\left(Y_{12 i, j} * \varepsilon_{j}\right)
$$

The cycle time $\left(\tau_{\max }\right)$ - generally one day - is also a constraint, in one cycle the expert visits the objects and return to his base location:

$$
\tau^{t}=\tau_{0,1}^{f}+\tau_{1}^{k}+\sum_{i=2}^{c^{t}}\left(\tau_{i}^{k}+\tau_{i-1, i}\right)+\tau_{q, 0}^{f}<\tau_{\max }
$$

where: $\tau^{t}$ : is the interval when the expert start from his base location, visit the objects and return, it is generally one day at the regional or countrywide maintenance systems and:

$$
\sum_{i=1}^{T} \tau_{i}^{t}=\vartheta
$$

where: (1) $T$ : is the number of cycles in the $\vartheta$ interval, (2) $\tau_{\text {max }}$ time interval of a cycle, (3) $c^{t}$ : the number of object have to visit in the cycle t, (4) $\tau_{i, 1}^{f}$ : the travel time to the first object, (5) $\tau_{q, 0}^{f}$ : the travel time from the last object to the experts base location, (6) $\tau_{i}^{k}$ : the average inspection time of the object $\mathrm{i}$.

The set of objects can be defined which have to inspect at the expert $\mathrm{c}$ :

$$
O_{c}:=\left\{o_{i} \mid Y_{12, i}=1 ; i=1 . . p\right\} ;\left|O_{c}\right|=P_{c},
$$

and the subsets, the objects have to be inspected in one cycle:

$$
O_{c}^{t} \subseteq O_{s},
$$

where: $O_{s}$ : is an ordered set, the objects assigned to the given expert, the ordering function is:

$$
o_{p} \in O_{i} ; o_{q} \in O_{i} ; o_{p}<o_{q} \text { ahol } t_{o_{p}}<t_{o_{q}}
$$

where: $t_{o_{p}}$ is the inspection time of $o_{p},(2) t_{o_{q}}$ is the inspection time of $o_{p}$, so the set is ordered by the visiting time.

$$
\begin{gathered}
\left|O_{c}^{t}\right|=c_{c}^{t} \leq P_{c}, \\
\bigcup_{t=1}^{T} O_{c}^{t}=O_{s} ; \bigcup_{s=1}^{p} O_{s}^{t}=O .
\end{gathered}
$$


However the expert perform more than one inspection on an object so the object is counted in the sets defined at (12) as many as the number of inspection has to be performed on it. To determine the interval of the inspections the following distance functions can apply:

$$
d\left(o_{i} ; o_{j} \mid o_{i} \in O_{p}^{t} ; o_{j} \in O_{q}^{t}\right)=p-q,
$$

so based on the constrain (4):

$$
\min \left\{d\left(o_{i} ; o_{j} \mid o_{i} \in O_{p}^{t} ; o_{j} \in O_{q}^{t}\right)\right\} \geq \tau_{i}^{m} .
$$

So the path travelled by the expert $\mathrm{i}$ in a cycle $\mathrm{t}$ can be describe as:

$$
l_{i}^{t}=l_{0, O_{i}^{t}(1)}+\sum_{c=1}^{\left|O_{i}^{t}\right|-1}\left(l_{o_{i}^{t}(c), o_{i}^{t}(c+1)}\right)+l_{O_{i}^{t}\left(\left|o_{i}^{t}\right|\right), 0}
$$

and the total path travelled by the expert $\mathrm{p}$ can be described as:

$$
l_{p}^{T}=\sum_{t=1}^{T}\left[l_{0, O_{i}^{t}(1)}+\sum_{c=1}^{\left|o_{i}^{t}\right|-1}\left(l_{O_{i}^{t}(c), O_{i}^{t}(c+1)}\right)+l_{O_{i}^{t}\left(\left|o_{i}^{t}\right|\right), 0}\right]=\sum_{t=1}^{T} l_{p}^{t}
$$

The expenditures $(\mathrm{C})$ of the experts $(\mathrm{S})$ in a given period $(\mathrm{T})$ can described as:

$$
C^{S}=\left[\sum_{j=1}^{S}\left(\sum_{t=1}^{T} l_{j}^{t}\right)\right] * c_{u}+\left[\sum_{j=1}^{S} P_{j}\right] * c_{v}
$$

where: (1) $c_{u}$ : is the specific cost for one kilometre, (2) $c_{v}$ : the specific cost for an object. The main target function is the minimal expenditures:

$$
C^{S} \rightarrow \min
$$

Unfortunately due to the limitation of this publication the whole model can't be presented here, but it is described in (Kota et al., 2002) but it has no impact on the optimisation process described in the next chapter.

\section{Literature on the multi-depot multiple travelling salesman problem}

The problem area of the technical inspection and maintenance systems discussed here is closest to the multiple travelling salesman problems (MTSP). And within the MTSP it is the fixed destination multiple depot multiple travelling salesman problem (MDMTSP), but the solutions in the literature did not mention the additional sub tour construction. This area is poorly researched compared to the general TSP or MTSP problems. Only the newest researches dealing with this field, and they utilize such heuristic techniques as: 
- agent based modelling with probability collectives: (Anand et al., 2010) developed a multi-agent model to solve the MTSP problem where they used collective memory, probability collectives, and stochastic methods, the algorithm use simple inserting, swap and elimination heuristics. They test the algorithm in two simple cases with fifteen nodes and three agents,

- genetic algorithm: (Suk-Tae Bae et al., 2007): They solve the problem with a two phase algorithm. In the first phase the problem was reduced from a multi centre problem into more single centre problems. In the second phase the problems solved individually with a genetic algorithm. The algorithm was tested up to 99 nodes and 4 agents,

- ant colony algorithm, (Ghafurian et al., 2011): They used the ant colony algorithm developed by Marco Dorigo (Dorigo, 2004) which is a wellresearched algorithm in these days (Crawford et al., 2009) and solve the general MmTSP model, where artificial ants search the solution in a problem tree modelled the foraging behaviour of real ants. The algorithm is compared by the solution of the Lingo 8 software and it can be seen that the ant colony heuristic algorithm is far faster and sometimes gives better solution. But the algorithm was tested only up to 40 nodes and 5 agents.

The studies of optimisation of complex logistics systems can be categorized into two main streams. The first stream addressed the application of meta-heuristic optimisation and the second stream focuses on the application of simulation. However simulation is a useful tool to optimise systems (Bányai, 2009), but the complexity of the system can extremely increase the required execution time, especially in the case of global, virtual systems (Bányai, 1999).

It can be seen that the developed methods was tested only a few nodes and they not includes the special constrains what emerge in technical inspection and maintenance system. But in the field of logistic there are systems with over 1000 nodes or rarely but systems with over 10000 nodes exist.

\section{Optimisation of the complex expert object assignment of a technical inspection and maintenance system with evolutionary programming}

\subsection{Evolutionary programming}

The problem-solving algorithms, which are a known mechanism of evolution are based on evolutionary algorithms are called. The most known algorithms are the: (1) genetic algorithm, (2) evolutionary programming, (3) evolution strategic, (4) neuro-evolution.

All the algorithms have a common part: they handle a population. The population is consisting of individuals. One individual is one possible solution to the problem. The target is to get the best solution to the given problem. But in the most of the problems the algorithms didn't have a chance the find the optimal solution and the one have to satisfy with a quasi-optimal or a "good enough" solution.

The evolutionary programming is mainly used on heavily constrained problems. This method is also handling a population but there are no limitations to the problem representation like at the genetic algorithm where bit vectors describe the 
individuals. Here the problems described as the problem allow, or it is the best for computer algorithms. The pseudo code of the evolutionary programming is the following:

1. generate the first population, in most cases it is random generated,

2. calculate the population fitness value

3. while not done

3.1.copy the population into a temporary population

3.2.run the mutation operators on the temporary population

3.3. select the survivors for the next population

4. end while

In the computer solutions first initialise the data, random generator, etc. Then initialise the first population. In heavily constrained problems there are two cases: (1) the random generated population individuals' are invalid: it violates the constrains, (2) the individuals are correct: but this is a very rare case.

There are several methods to get valid individuals from simply dispose invalid individuals to create special operators which retain the individual's integrity. But the simplest solution is using penalty functions. In the penalty functions one can regulate the algorithm which solutions are preferable.

After the creation of the initial population it have to copied into a temporary population then the mutation operators run on the temporary population. In most cases the high impact mutations have a lesser chance to run and the low impact operators have a bigger chance. After the mutation we have to compute the mutated individuals' fitness value and then choose the survivor individuals to the descendant population which happen with a championship. One simple way to perform the championship is choose two random individuals one from the original and one from the mutated population and that will survive which has a lesser (or bigger if the fitness not normalized) fitness value, we have to repeat this until the new population not filled.

At the evolutionary programming the vast majority of the cases there are no crossover. In some solutions there is no meaning of this, and mostly it creates invalid individuals. So in the biological view this is not an evolution of one species but the evolution of many so calling it "population" is not valid but for the integrity of evolutionary method this naming convention is common.

\subsection{The problem representation in the proposed evolutionary programming algorithm}

The developed algorithm solve the fixed destination multiple depot multiple travelling salesman problem with sub tours and optimise the salesman count in one phase and can be used on large or very large problems. As there are multiple salesman: the experts, multiple depot: all the experts have different locations, fixed destination: all the expert start and return to their initial location, and all the experts do the travel in (generally) one day cycles. And crowns all it regards

The developed solution method based on a multi chromosome technique which is not widely used in genetic algorithm but it could simply implement in the evolutionary programming. 
The data structure of the optimisation is built as describe in the 4.1. The biggest container is the population which consists of defined constant number of individuals, which is an input parameter of the optimisation

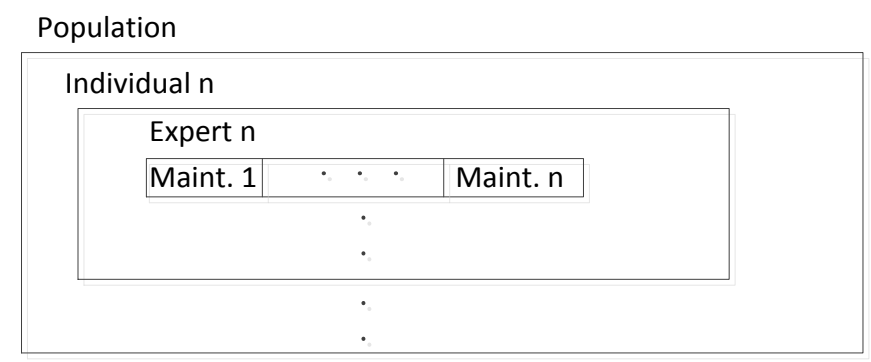

Fig. 3. The cascaded data structure of the optimisation

First the algorithm initializes the optimisation constants, like penalty constants, maximum tour length, maximum cycle count, expert performance values and number of the individuals in a population, load the data files of the experts, that's define the number of the experts and their location the objects which define the number of the objects and its location, and initialize maintenances data. Then it creates the first population with random generated individuals. It is a top down algorithm: (1) first generate individuals container, (2) then generate experts container and insert it into the individuals, (3) then generate chromosome and insert it into the experts so that fill the chromosome with maintenances in a random generated order. So every expert is a chromosome that's why the algorithm named as multi chromosome algorithm. Then it calculates the fitness values of the experts and applies the penalty functions for the experts, finally apply the global penalty functions for the individual.

\subsection{Penalty functions}

The penalty functions is one of the simplest and fastest way to rate the individual, so the goodness of the actual solution. In this algorithm there are (1) local - the penalty function is applied to the expert - and (2) global - the penalty function is applied to the whole individual -

penalty functions applied.

\subsubsection{Local penalties}

There are four different local penalty functions:

- Cycle count penalty: when the expert do more route cycles as the allowed(5),

- Few penalty : the expert has to get a minimal number of maintenances (8),

- More penalty: the expert cannot get more maintenances than his maximum capacity (8),

- Near penalty: the maintenances of one object cannot be arbitrarily close (4). Cycle count penalty function of the expert $\mathrm{i}$ :

$$
P_{C}^{I}=P_{C C} * c_{i}
$$


where:

- $P_{C C}:$ constant, the penalty value of the cycle count violation

$-c_{i}$ : the actual number of the expert's cycles.

Few penalty function of the expert $\mathrm{i}$ :

$$
P_{F}^{I}=P_{F P} *\left(P_{\text {min }}-P_{i}\right)
$$

where:

$-P_{F P}$ : constant, the penalty value of the few maintenances violation

- $P_{i}$ : the actual performance of the expert $\mathrm{i}$, the number of the maintenances

The more penalty function of the expert $\mathrm{i}$ :

$$
P_{M}^{I}=P_{M P} *\left(P_{i}-P_{\max }\right)
$$

where: (1) $P_{M P}$ : constant, the penalty value of the more maintenances violation (2) $P_{i}$ : the actual performance of the expert $\mathrm{i}$, the number of the maintenances

The near penalty function is the following:

$$
\begin{aligned}
& \text { if }\left[M_{i}^{x}\right]-\left[M_{i+1}^{x}\right]<\tau^{m} \text { then } c_{N}=c_{N}+1 \text {; } \\
& P_{N}^{I}=\sum_{1}^{s} P_{N P} * c_{N}
\end{aligned}
$$

where: (1) $M_{i}^{x}$ : the maintenance $\mathrm{i}$ of the object x, (2) $P_{N P}$ : constant, the penalty value of the near maintenances violation, (3) []: indexof operator, show the index of the given maintenance, (4) $c_{N}$ : the number of the maintenance near violations.

\subsubsection{Global penalties}

There are two different global penalty functions, which calculated after the local penalties: (1) Scatter penalty: which applied when an object's maintenances are scattered among several experts (chapter 4.2), (2) Expert count penalty: The expert's employment have a fixed cost in this model. Due to this penalty functions the algorithm try to minimize the number of employed experts.

Scatter penalty function is the following:

$$
\begin{gathered}
\text { if } \operatorname{count}\left(M_{i}^{x}\right)<\operatorname{required}\left(M_{i}\right) \text { then } c_{P}=c_{P}+1 \\
P_{S}=\sum_{1}^{s} P_{S C} * c_{P}
\end{gathered}
$$

where: (1) $\operatorname{count}\left(M_{i}^{x}\right)$ : is the count of the maintenances of the object $\mathrm{i}$ at the expert $\mathrm{x}$ (2) required $\left(M_{i}\right)$ : the required maintenances of the object I (3) $P_{S C}$ : constant, the penalty value of scattered maintenances. 
Expert count penalty function:

$$
P_{E}=P_{E C} * \mathrm{~s}
$$

where: (1) $P_{E C}$ : constant, the cost of one experts' employment, (2) $s$ : the number of the experts.

Then the algorithm enters into the optimisations' main loop (chapter 6.1) and copies the population individuals into a temporary population in order of their fitness so the best fitness value individual is copied into the first position of the temporary population. The applied operators in the algorithm are simple and more or less common at the genetic methods, because more the operators are simpler the faster the algorithm.

\subsection{Mutation operators}

In the mutation phase the algorithm mutates the individuals in the temporary population. There are two types of mutation operators due to the multi chromosome characteristic: (1) inner expert mutation operators and (2) cross expert mutation operators.

\subsubsection{Inner expert mutation}

There are three types of inner expert mutation operators:

- Gene swap: where two random genes are swapped:

$$
-
$$

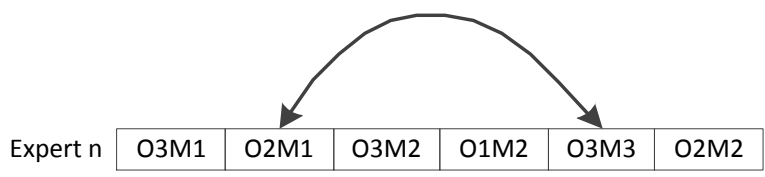

Fig. 4. Gene swap operator

- Gene sequence reversion: where the gene sequence are reversed between two random indexes

Fig. 5. Gene reversion operator

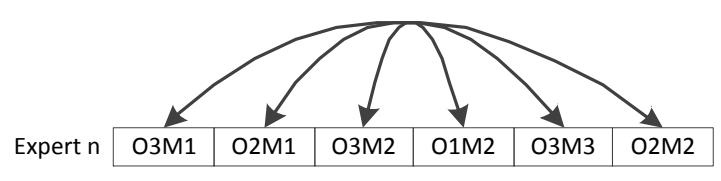

- Gene insertion operator: where a randomly chosen gene inserted into a randomly chosen position

Fig. 6. Gene insertion

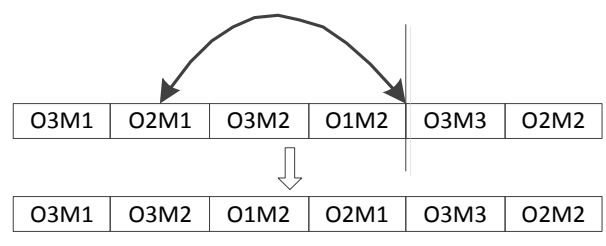




\subsubsection{Cross expert mutation}

Like the inner expert mutation operators there are also three types of mutation operators exists for the cross expert mutation. These are the followings:

- Cross expert gene swap: which swaps two genes between experts. The second expert and the position of the second gene are chosen randomly.

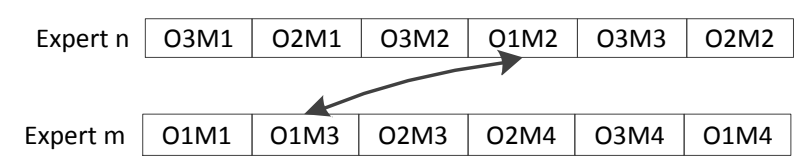

Fig. 7. Cross expert gene swap

- Cross expert gene sequence change: where the algorithm swapping a randomly chosen but continuous gene sequence with a randomly chosen expert also randomly chosen gene sequence:

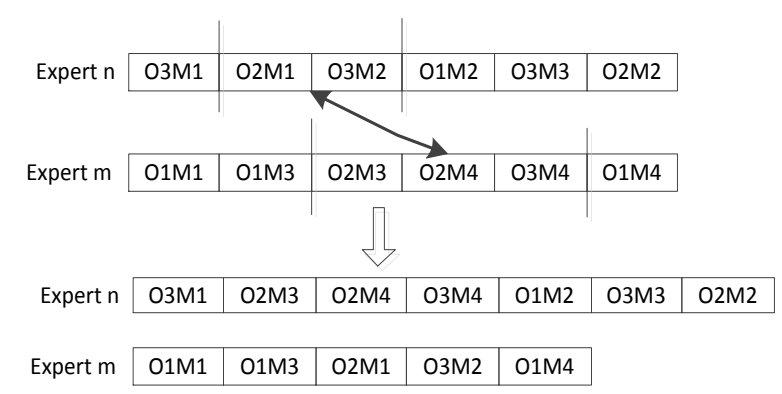

Fig. 8. Cross expert gene sequence swap

- Cross expert gene contraction: where random amount of genes inserted to a randomly chosen expert from the end of the chromosome

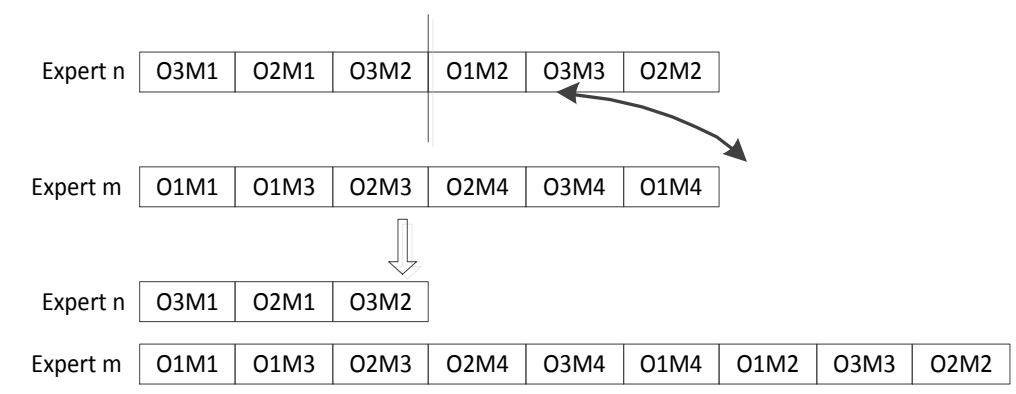

Fig. 9. Cross expert chromosome contraction

The mutations are chosen randomly for every expert with parameterised probability and the algorithm allows a probabilistic value for the no mutation also.

\subsection{Survivor selection}

First the algorithm search for the best individual and copy it to the descendant population. Its called elitism, the best individual (elitist) always survives. The evolutionary programming typically uses stochastic tournament survivor selection. The simplest of the tournament selection is when randomly choose two individuals, one from the original and one from the mutated population and the fittest win, that individual will be copied into the next generation of the individual. This process is repeated until the next population is filled. 
Kota, L.: Optimisation of Large Scale Maintenance Networks with Evolutionary...

But for avoiding the local optimums all of the genetic methods trying to maintain the diversity among the individuals, in this algorithm one can parameterise how much individual in the descendant population will be filled with random generated individuals

The main loop of the optimisation now stops on an exact repetition count.

\section{Results}

The algorithm was tested on several tsp files from the de facto standard TSPLIB sample instances with random experts' locations. The algorithm gives very good run times on large $(1000-2000$ objects and 30-50 experts) instances. It is tested on a very large instance over 11000 objects but the running time with the current implementation is a bit long, about $2-3$ weeks on a $2.9 \mathrm{Ghz}$ I7 machine using a single core.

Convergence with the tsplib att48.tsp file:

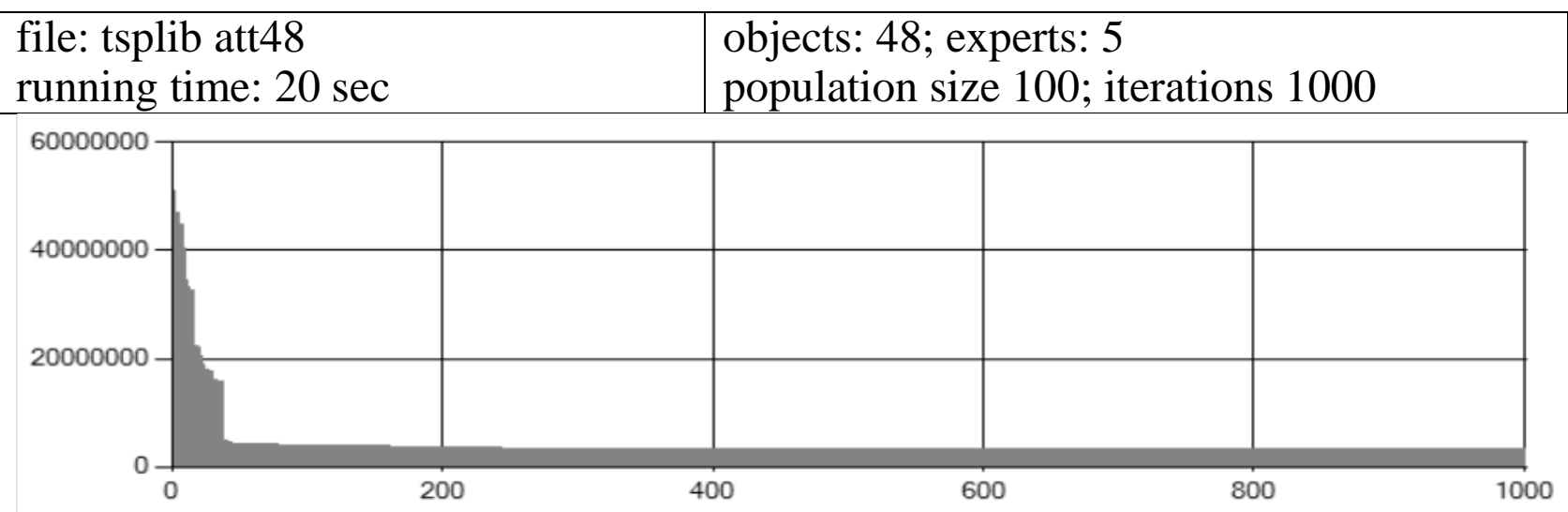

Tab 1. tsplib att48

As the diagram shows, the algorithm convergences very fast in this simple instance, at the first 100 iterations and after then get slower up to the 400 iteration at this simple instance and then no further improvements are visible. But this is usual in the field of genetic methods

Convergence with the tsplib pr654 file:

\begin{tabular}{|l|l|l|}
\hline $\begin{array}{l}\text { file: tsplib pr654 } \\
\text { running time: } 29 \min 30 \mathrm{sec}\end{array}$ & $\begin{array}{l}\text { objects: 654; experts: 30 } \\
\text { population size 100; } \\
\text { iterations 1500 }\end{array}$ \\
\hline 600000000 & & \\
\hline 400000000
\end{tabular}

Tab. 2. tsplib pr654 
At this instance the diagram shows very fast convergence up to 600 iterations and after then only minor improvement happened. This instance with the 654 nodes counts a medium size instance in the field of logistics.

Convergence with the tsplib pr1002file:

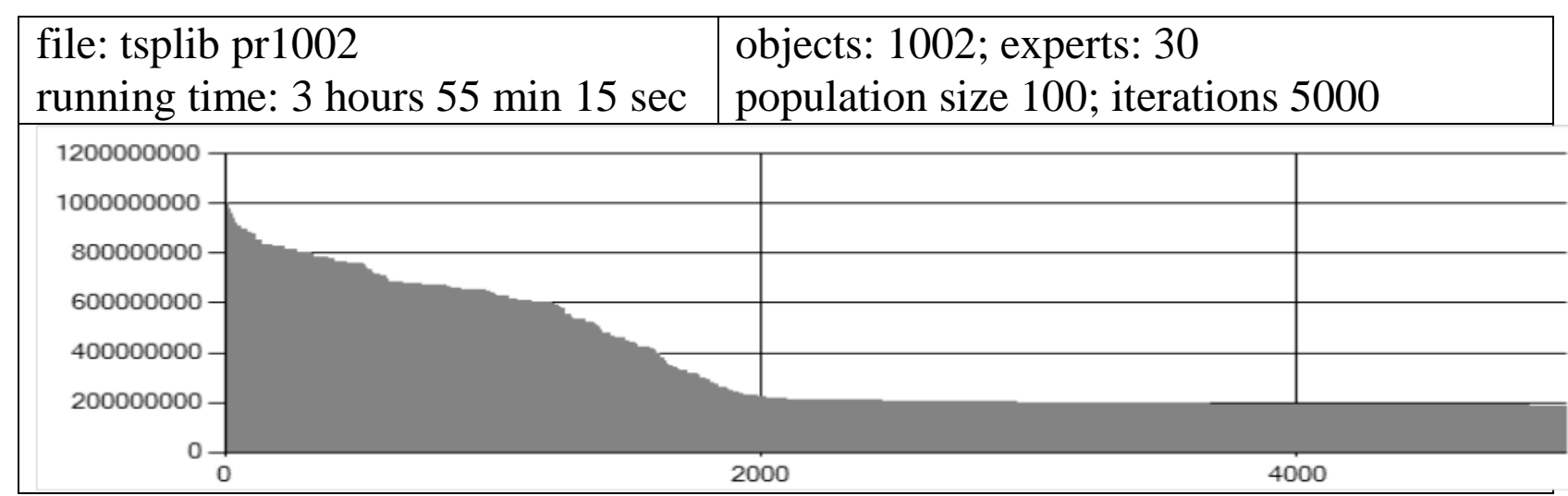

Tab 3. tsplib pr1002

At this large instance the convergence a bit worse than the previous instances. As it can be seen the target function improves fast up to the 2000 iteration count and after that only minor improvements can be observed.

As it can be seen that, the execution time is increasing as the problem size grows, but it is stay in manageable range even in this large problem size. But a little over this size the further improvement of the implementation of the algorithm requires parallelization or other performance increase method.

\section{Further researches}

The optimisation method has great potentials toward the further improvements. First some improvement in the method himself:

- try new mutation methods,

- try new survivor tournament selection methods.

Second some improvements which have a great impact on speed:

- parallelization: some current researches which is not ready for publications a simple parallelization of fitness calculation can improve the running time by reducing it to the third in large scale problems, but in small scale the task switching and data moving processes slower the calculation.

- examine the implementation possibilities on a fast graphics processing unit.

\section{Acknowledgements}

The research was supported by the TÁMOP 4.2.1.B-10/2/KONV entitled Increasing the quality of higher education through the development of research development and innovation program at the University of Miskolc. 
Kota, L.: Optimisation of Large Scale Maintenance Networks with Evolutionary...

\section{References}

Anand J. Kulkarni, K. Tai (2010): Probability Collectives: A multi-agent approach for solving combinatorial optimization problems, Applied Soft Computing 10, pp.: 759-771, doi:10.1016/j.asoc.2009.09.006

Bányai, Á. (1999) Das virtuelle Logistikzentrum als Koordinator der logistischen Aufgaben. In: Modelling and optimization of logistic systems - Theory and practice, Bányai, T. \& Cselényi, J. (Eds.), pp. 42-50, ISBN 963661402 4, Published by the University Miskolc

Bányai, T. (2009): Optimisation of U-shaped flexible manufacturing cells. In: Annals of DAAAM for 2009 \& Proceedings of the 20th International DAAAM Symposium "Intelligent Manufacturing \& Automation: Focus on Theory, Practice and Education", Katalinic, B. (Ed.), pp. 761-762, ISSN 1726-9679, ISBN 978-3-901509-70-4, Vienna, Austria, November 2009, Published by DAAAM International Vienna

Crawford, B.; Castro, C. \& Monfroy, E. (2009): Enhancing the Transition Rule in Ant Computing for the Set Covering Problem, Annals of DAAAM for 2009 \& Proceedings of the 20th International DAAAM Symposium, 25-28th November 2009, Vienna, Austria, ISSN 1726-9679, ISBN 978-3-901509-70-4, Katalinic, B. (Ed.), pp. 1329-1330, Published by DAAAM International Vienna, Vienna

Dorigo, M., Stützle T. (2004): Ant Colony Optimization, MIT Press. ISBN 0-26204219-3

Kota, L., Cselényi, J. (2002): Defining the new experts location in an elevator maintenance examination system, "Вестник Национального технического Университета «ХПИ» 9'2002 т. 10, Харьков, Сборник научных трудов Тематический выпуск” “Технологии в машиностроении“ Удк 621.9, страницы:96-102

Ghafurian, S., Javadian, N. (2011): An ant colony algorithm for solving fixed destination multi-depot multiple traveling salesmen problems, Applied Soft Computing 11, pp.: 1256-1262

Suk-Tae, B., Heung Suk, H., Gyu-Sung, C. and Meng-Jong G. (2007): Integrated GA-VRP solver for multi-depot system, Computers \& Industrial Engineering Volume 53, Issue 2, Pages 233-240, doi:10.1016/j.cie.2007.06.014 